\title{
Neue Herausforderungen
}

\author{
Barbara Schieche
}

Die Wohlfahrtsverbände sind auf der Suche nach ihrer Rolle in einer sich rasch verändernden Umwelt. Einerseits werden sie von ihren Mitgliedsorganisationen als Unternehmensverband gefordert, andererseits sehen sie sich aus aufgrund ihres gesellschaftlichen Auftrags als Anwalt hilfesuchender Menschen. Ein Expertenforum suchte nach Antworten, wie sich die Verbände neu positionieren können.

Ende letzten Jahres feierte Dr. Eva Douma, die als Beraterin für Unternehmen der Sozialwirtschaft spezialisiert ist, ihr zehnjähriges Firmenjubiläum mit einem Expertenforum zum Thema »Sozialwirtschaft im Wandel«. Dort berichtete die Gastgeberin von ihren Erfahrungen aus zehn Jahren Beratung für soziale Institutionen und ließ jeweils einen Vertreter aus Politik und der Sozialwirtschaft zu Wort kommen: Prof. Erika Fellner, Hochschullehrerin für Sozialarbeit und ehemalige Landtagsabgeordnete in Hessen, und Harold Becker vom Paritätischen Wohlfahrtsverband aus Kassel.

Das Ergebnis war eine lebhafte Diskussion über die $\mathrm{Zu}$ kunft der Wohlfahrtsverbände. So unterschiedlich die Erfahrungen und Denkansätze, so einhellig war das Fazit: Die Wohlfahrtsverbände stehen derzeit in einer harten Umbruchphase und müssen sich neu definieren. Hier gilt es einerseits den Herausforderungen des Marktes zu begegnen und andererseits ihrer Rolle als Anwalt der Schwachen gerecht zu werden.

Wie hat sich die Sozialwirtschaft in den vergangenen zehn Jahren verändert? Für Eva Douma zeichnet sich eine Entwicklung klar ab: Soziale Dienstleistungen werden in $\mathrm{Zu}$ kunft zunehmend von privaten Dienstleistern angeboten. Über kurz oder lang wird es ihrer Meinung nach keinen Wohlfahrtsbonus mehr geben: »Wenn es um praktische Fragen der Organisationsstruktur geht, macht es keinen Unterschied, ob ein Unternehmen gewinnorientiert arbeitet oder nicht oder ob es - wie bei den Wohlfahrtsverbänden eine Zwitterstellung einnimmt. Anders formuliert: Ich sehe keine Notwendigkeit, im Hinblick auf die interne Organisationsstruktur in der Wohlfahrt anders zu arbeiten als in einem normalen gewinnorientierten Betrieb.«

Früher war die Arbeit für einen guten Zweck - vom Staat gewollt und bezahlt - ein klares Spezifikum der Wohlfahrt. Für die Finanzierung war vor allem entscheidend, dass ein politisch überzeugendes Konzept zur Versorgung bestimmter Zielgruppen vorlag. Heute fragt dagegen keiner mehr nach politischen Konzepten. Vielmehr werden unangenehme Fragen gestellt wie: Was bringt das? Was kostet das? Wieso geht das nicht billiger? Damit rücken Themen wie Organisationsstruktur und Personalführung in den Fokus, denn jetzt heißt es, seine Arbeit als Wohlfahrtsverband wirtschaftlich zu legitimieren und sich zu reorganisieren.

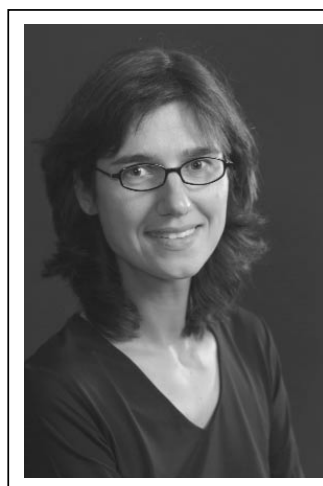

Barbara Schieche ist Kommunikationsberaterin und Journalistin mit einem eigenen Büro in München. Sie hat an der Hochschule der Jesuiten in München Philosophie studiert und eine Fortbildung über systemische Beratung absolviert. Nach einiger Zeit in verschiedenen Positionen in Redaktionen und Verlagsleitungen ist sie seit über zehn Jahren selbstständig tätig. Internet http://www.schieche-genau.de

\section{Beispiel Pflege - heute zunehmend in privater Hand}

Stand heute ist laut Eva Douma: Sozialwirtschaftliche Leistungen, deren privatwirtschaftliche Erbringung vor Jahren noch undenkbar gewesen wäre, werden mittlerweile von privatwirtschaftlichen und gewinnorientierten Unternehmen angeboten. Besonders deutlich wird dies in der Pflege. Dort existieren mittlerweile unzählige private Dienstleister, während kommunale Anbieter fast völlig verschwunden sind - sie wurden geschlossen oder durch private Träger übernommen. Zwar gibt es noch Pflegeeinrichtungen der Wohlfahrt, die jedoch in diesem Segment kaum expandieren. Die Tendenz geht hier eher zur Konzentration und Stagnation.

Was die Pflegeleistungen für die Kunden betrifft, gibt es durchaus die privaten Anbieter, die mit hoher Professionalität arbeiten: »Meiner Erfahrung nach sind in der Pflege nicht sehr viele Einrichtungen zu finden, die Spitzenqualität bieten. Dort, wo dies der Fall ist, müssen sich private Anbieter nicht verstecken. Im breiten Mittelfeld des Leistungsangebotes dominiert der große Block der Wohlfahrt und ganz unten gibt es Kleineinrichtungen, die manchmal hart am Rande der Illegalität arbeiten hier finden sich viele Private.«

Kurzum: Pflegeleistungen können von den Privaten hochprofessionell und in bester Qualität erbracht werden und es ist mitnichten so, dass die privaten Anbieter den Gewinn und die Wohlfahrt den Menschen in den Mittelpunkt stellen. Im Gegenteil: Klientenbefragungen von privaten Pflegeanbietern zeigen eine große Kundenzufriedenheit - die Klienten fühlen sich von den privaten Dienstleistern bestens um- und versorgt. Den Unterschied zwischen den "guten Diensten der Wohlfahrt « und den »bösen, allein aufs Geld fixierten Privaten« gibt es nicht. 
Die Prognose von Eva Douma ist daher folgende: »Ich behaupte, dass die Pflegeversicherung der Einstieg in den Ausstieg war, was die Anbieterstrukturen betrifft. Ich prognostiziere, dass wir in zehn Jahren in noch wesentlich mehr Bereichen der Sozialen Arbeit private Anbieter haben werden. Private Kindergärten, private Betreuer, private Behinderteneinrichtungen, private Jugendhilfe - in Ansätzen ist das alles schon da. Und die Einführung des Persönlichen Budgets sehe ich hier als einen Katalysator. Da werden sich gemeinnützige Einrichtungen noch umschauen müssen und es dürfte in allen Bereichen solche Eruptionen geben, wie in der Pflege schon geschehen.«

\section{Trend: Wohlfahrt reorganisiert sich}

Freilich sind den Non-Profit-Unternehmen die Aktivitäten der privaten Anbieter nicht verborgen geblieben. So haben die gemeinnützigen Einrichtungen verstanden, dass es etwas bisher nie Dagewesenes gibt: Mitbewerber und Konkurrenz. Die Reaktionen darauf war eine Professionalisierung der Organisationsstruktur, wobei manche Wohlfahrtsverbände hier übers Ziel hinausschossen und zur großen Verwunderung und Irritation ihrer Klienten und Förderer hochpreisige Beratungsunternehmen wie Roland Berger oder McKinsey engagierten. Diese auf Wirtschaftsunternehmen spezialisierten Beratungshäuser verstanden jedoch nicht, dass sich ein Wohlfahrtsverband nicht wie ein gewinnorientierter Konzern durch drastischen Personalabbau »verschlanken« lässt. So wurde hier viel Geld in eine Beratung investiert, die am Ende nicht zu den gewünschten Ergebnissen führen konnte.

Einige Verbände haben es inzwischen geschafft, professionelle Organisationsstrukturen zu etablieren: klare Kompetenzen, exakt definierte Aufgaben, detaillierte Zielvorgaben, nicht zu vergessen die Festlegung von zur Verfügung stehenden Ressourcen. Tendenz steigend - Professionalisierungsbestrebungen sind fast überall in der Wohlfahrt erkennbar.

\section{Personalführung: in Wohlfahrtseinrichtungen nicht besser als in der Privatwirtschaft}

Große Probleme bereitet den Sozialen jedoch die Mitarbeiterführung, wie Eva Douma zu berichten weiß: »Da gibt es eine soziale Einrichtung mit heftigen Finanzproblemen, weil der Buchhalter leider die Buchführung nicht beherrscht. Jetzt könnte man sagen: Ja, aber wir sind doch ein Betrieb der Behindertenhilfe und haben in unserer Buchhaltung immerhin einen Arbeitsplatz geschaffen, da können wir doch nicht so harte Maßstäbe anlegen! Nur im konkreten Fall war die Buchführungskraft überhaupt nicht gehandicapt, sondern einfach unfähig und obendrein auch noch bildungsresistent. Dennoch wurde nicht die entsprechende Konsequenz gezogen. Aber ist es sozial, unwillige Mitarbeiter zu beschäftigen und sie mit Geldern zu bezahlen, die man von der öffentlichen Hand erhalten hat?«

Die Erfahrung zeigt, dass Personalführung in den Einrichtungen der Wohlfahrt nicht besser oder »ethischer « ist als in der Privatwirtschaft. Zwar gibt es in der Wohlfahrt eine höhere moralische Anforderung an die Mitarbeiter - es soll mehr und engagierter gearbeitet werden - dafür erhal- ten die Angestellten jedoch eine schlechtere Bezahlung, weniger Förderung und oft eine mangelhafte Ausstattung. Und ein stärkeres Agieren auf der Beziehungsebene macht einen Arbeitsplatz nicht unbedingt mitarbeiterfreundlicher, im Gegenteil: Etwas mehr Klarheit und Rationalität würden die Arbeitsergebnisse verbessern und die Mitarbeiter entlasten - und längerfristig sogar weniger kosten.

\section{»Der Einsatz für hilfesuchende Menschen ist wieder gefragt"}

Doch die härtesten und persönlichsten Kämpfe im Umgang mit dem Personal werden ausgerechnet immer wieder bei den Wohlfahrtseinrichtungen ausgetragen, so zumindest die Erfahrung von Eva Douma: »Da wurden bei einem Dienst der Wohlfahrt jährlich zugewiesenen öffentlichen Finanzzuschüsse nicht mehr bewilligt. Dummerweise hatte die Einrichtung damit ihre Mitarbeiter bezahlt. Jetzt wurde erwartet, dass die Mitarbeiter im Sinne des Projektes gefälligst umgehend von der Bildfläche verschwinden und bitte nicht auf die Einhaltung von Kündigungsfristen bestehen, weil ja schließlich jede weitere Lohnzahlung das gemeinsame Projekt gefährden würde. Wer sich hier auf die Einhaltung arbeitsrechtlicher Regelungen berief, wurde als moralisch minder und unsolidarisch beschimpft. Da geht es doch bei so einer vermeintlich knallhart gewinnorientiert agierenden Institution geradezu integer zu: Die setzen zwar ihre Leute auf die Straße, wenn es sich nicht mehr rechnet. Aber es geht wenigstens nur ums Geld. Bestenfalls gibt es eine Abfindung. Kündigungsfristen werden eingehalten und ein Zeugnis, was die bisher gute Arbeit bescheinigt, wird auch zeitnah erstellt. Das sind wenigstens klare Verhältnisse.«

\section{Problem: Das »richtige« Personal finden}

In der breiten Öffentlichkeit hält sich die Meinung, dass Wohlfahrtseinrichtungen auch als Arbeitgeber sozial sein sollten. Hier, so die Einschätzung der Allgemeinheit, werde "ganz anders « gearbeitet als bei einer privaten gewinnorientierten Institution. Dieses Vorurteil hat für die Wohlfahrt

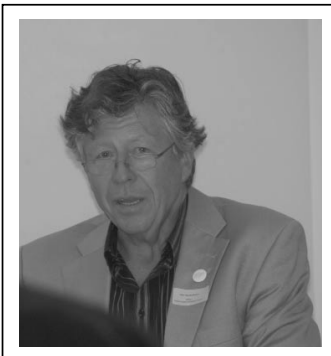

»Auf dem Hintergrund der großen Bedeutung der Dienstleistungsfunktion der Wohlfahrtsverbände müssen sie ihre marktfähigen und nicht-marktfähigen Bereiche deutlicher trennen und Umverteilungen transparent machen. Gleichzeitig müssen sie lernen, neben ihrer Rolle als korporatistisch agierende Sozialanwälte viel stärker die Rolle aktiv streitbarer sozialpolitischer Akteure der Entwicklung der Zivilgesellschaft einzunehmen.«

»Der Wandel des deutschen Sozialstaates hin zu einem >Fürsorge-, Almosen- und Suppenküchenstaat mit halbfeudalen (Unter-) Versorgungsstrukturen und in der Folge der rapide Bedarf an nichtmarktfähigen sozialen Dienstleistungen jenseits des Wohlfahrtsmarktes stellt die Wohlfahrtsverbände vor die Aufgabe, ihre Arbeit auf diesem Feld zu verstärken.«

Harold Becker, Geschäftsführer der Kreisgruppe Kassel des Paritätischen Hessen e. $V$. 
eine deutlich spürbare, negative Konsequenz: Ein ganz bestimmter Typus Mensch bewirbt sich eben nicht bei ihnen, obwohl er dringend gebraucht würde. So machen kirchliche Sozialstationen immer wieder die Erfahrung, dass sich bei ihnen kaum junge, belastbare Pflegekräfte bewerben - und gerade in der Pflege braucht es für die harte körperliche Arbeit junge Leute. Die nachwachsende Generation ist jedoch deutlich geringer kirchlich sozialisiert, ein vermeintlicher „Gutmenschen-Anspruch «schreckt junge Leute eher ab, weshalb sie lieber zu den privaten Diensten gehen, die eben keinen »moralischen Überbau« haben. Auch bei der Vergütung sind wohlfahrtliche Einrichtungen ins Hintertreffen geraten. Denn es gibt dort keinen Bonus der Kostenträger für Wohlfahrtsverbände mehr.

Ein weiteres Problem für Wohlfahrtsverbände liegt darin, Leitungskräfte mit betriebswirtschaftlichem Knowhow zu finden, die sich nicht nur mit den spezifischen Wohlfahrtsstrukturen auskennen, sondern sich auch noch mit dem jeweiligen Leitbild identifizieren können. Abgesehen von der oftmals schlechteren Bezahlung, ist es für Arbeitnehmer, die nicht aus dem sozialpädagogischen Bereich kommen, nicht immer leicht, sich in die interne Sozialkultur einzufügen beziehungsweise dort aufgrund eines anderen Habitus akzeptiert zu werden. Damit gibt es bis heute zu wenig ökonomisches und juristisches Knowhow in den Verbänden, auch in den großen Einrichtungen, wie Eva Douma weiß: »Da erstellt dann der Sozialarbeiter die Bilanz und so sieht die dann auch aus.«

Gut qualifizierte Fach- und Führungskräfte wissen heute, dass ihnen die privaten Dienste nicht nur interessante Aufgaben und Entwicklungsperspektiven bieten, sondern dass gerade bei den privaten Anbietern auf die Work-Life-Balance geachtet wird. So berichtet Eva Douma aus ihrer Erfahrung: "Ich kenne viele wunderbar geführte private Einrichtungen, die eine soziale und verantwortungsvolle Personalführung haben. Was tun, wenn die Pflegekräfte jung sind und kleine Kinder haben, die während der Dienstzeiten betreut werden wollen? Nein, nicht kinderlose Mitarbeiterinnen suchen, sondern: eine Tagesmutter für die Kinder der Mitarbeiterinnen einstellen. Eigentlich eine ganz naheliegende Lösung.«

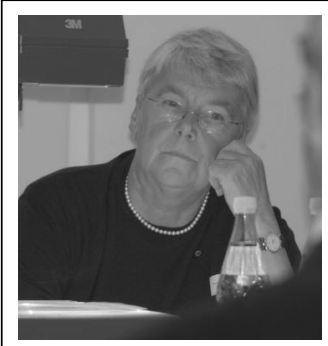

»Staatliche Sozialpolitik setzt vermehrt auf die Förderung wettbewerblicher Strukturen. Es ist Vergangenheit, dass Wettbewerb im sozialen Bereich als ungeeignet gilt, weil dies zulasten der Hilfsbedürftigen gehen könnte. Heute wird nur der Frage nachgegangen, wie der Wettbewerb sozialer Dienstleistungen so gestaltet werden kann, dass er Wahlmöglichkeiten für Nutzer schafft, qualitativ gute Dienstleistungen ermöglicht und Leistungen zu angemessenen Kosten erbringt. Die freien Verbände werden mit Vorwürfen mangelnder Transparenz, ineffektiver Leistungen und Bildung von Kartellen konfrontiert.«

»Kostensenkung und Budgetkontrolle verstärken sich in der Sozialpolitik. Subsidiarität wird vor allem als Eigenverantwortung beim Individuum gesehen. Das System sozialer Dienste erhält damit größere wirtschaftliche und arbeitsmarktpolitische Bedeutung. Einmal geht es um Leistungsvereinbarungen und dann im Rahmen von Modernisierungsprozessen der freien Verbände um die Trennung von Verband und Dienstleistungen. Die Entwicklung ist nicht erst im Kommen, sie hat längst begonnen.«

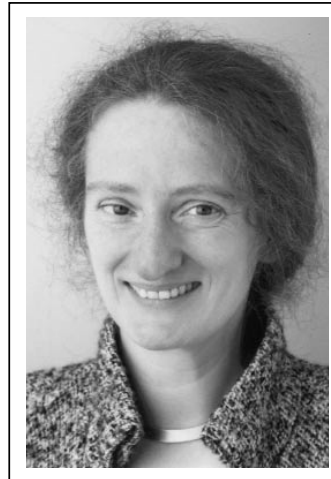

Dr. Eva Douma studierte Sozial- und Verwaltungswissenschaften an der Universität Bielefeld und der Hochschule für Verwaltungswissenschaften in Speyer. Gefördert als Stipendiatin der Volkswagenstiftung promovierte sie zu einem rechtsgeschichtlichen Thema. Seit 1993 ist sie Lehrbeauftragte an verschiedenen Fachhochschulen und Akademien, u. a. Fachhochschule Darmstadt am Fachbereich Wirtschaft, Steinbeis-Akademie und Hessische Verwaltungs- und Wirtschafts-Akademie Frankfurt am Main. Nach einer Referententätigkeit beim Paritätischen Landesverband Hessen machte sie sich 1997 als freiberufliche Organisationsberaterin und Trainerin selbstständig. Ihr Arbeitsschwerpunkt liegt in der Bearbeitung von Organisations- und Personalfragen insbesondere für Dienste des Gesundheits- und Sozialwesens.

Internet http://www.douma.de

\section{Rückbesinnung der Wohlfahrtsverbände auf ihre Rolle als Anwalt der Schwachen}

Was sollten die Wohlfahrtsverbände tun, um die derzeitigen Probleme in Organisation, Personalrekrutierung und Mitarbeiterführung zu lösen? Als erstes ist es sicherlich geboten, aufzuhören darüber zu klagen, dass ihr Einfluss im Sozialbereich zunehmend geringer wird. Vielmehr sollten sich die Wohlfahrtsverbände wieder auf ihr Alleinstellungsmerkmal besinnen: Lobbyist der Schwachen in dieser Gesellschaft zu sein. Eine Chance dazu wurde leider verpasst: Bei der Einführung der sogenannten Hartz IV-Reform, die im SGB II ihren Niederschlag gefunden hat, gab es zwar Stellungnahmen der Verbände, aber kein echtes Engagement für die betroffenen Menschen. Aber nicht nur, dass die Wohlfahrtsverbände nicht geschlossen gegen diese neue Ungerechtigkeit aufgestanden wären - sie haben sich obendrein auch noch zu »Handlagern« der Arbeitsagenturen gemacht. Denn nach $\S 16$ SGB II wird der Wohlfahrtsverband eingeschaltet, um als Dienstleister der Arbeitsagentur einen Teil der entsprechenden Eingliederungsvereinbarung abzuarbeiten und als Dienstleister unterliegen sie dem Controlling des Fallmanagers. Allein damit wurde ein Stück Glaubwürdigkeit in der Öffentlichkeit und bei der Klientel verspielt und auch die Rolle gegenüber der Politik geschwächt.

Hier müssen die Wohlfahrtsverbände aktiv gegensteuern. Deshalb fordert Eva Douma: »Wenn die Wohlfahrt als Institution überleben will, dann muss sie im politischen Bereich wieder stärker agieren. Die letzten zehn Jahre haben sich die Einrichtungen und Verbände stark darauf konzentriert, ihre internen Strukturen und ihre Leistungen zu optimieren. Beides war und ist notwendig. Denn die Wohlfahrtsverbände brauchen gute Leistungen und eine gute interne Organisations- und Personalführung, um auf dem Markt der Sozialwirtschaft überleben zu können. Aber das macht die Wohlfahrt nicht aus. Plakativ formuliert: Wenn die Wohlfahrt sich nicht auf ihre ursprüngliche Rolle als Anwalt der Schwachen besinnt und gegenüber der Politik genau in dieser Rolle mit klaren Positionen und Forderungen auftritt und sich nur noch als Sozialdienstleister versteht, dann brauchen wir keine Wohlfahrt mehr. Denn die Erbringung von Dienstleistungen gegen Entgelte kann auch vollständig privat gestaltet werden. 